\title{
Mejora de la implicación del alumnado en "Diseño y síntesis de sistemas digitales" usando e-learning colaborativo, gamificación y aprendizaje basado en problemas
}

\section{Oscar Alonso $^{\text {a }}$, Jordi Colomer ${ }^{\mathrm{a}}$, Nil Franch ${ }^{\mathrm{a}}$ y Angel Diéguez ${ }^{\mathrm{a}}$}

åDepartamento de Inenierías: Sección Electrónica, Universitad de Barcelona, oalonso@el.ub.edu

\begin{abstract}
During the last 3 years, it has been observed that the students do not have a solid base to understand the concepts that are introduced in the subject. In addition, the attitude of the students, in general, is negligence, do not review concepts to prepare the subject, do not prepare laboratory practices and stop attending class. This results in a high dropout rate, fear of doing the subject and discomfort with the teaching staff.

To solve this problem, we apply the teaching innovation presented in this article. Such innovation takes advantage of teaching strategies such as gamification or problem-based learning, uses virtual tools to motivate elearning and collaboration with other students. The results, to date, are satisfactory since they reduce the dropout rate and increase the number of students who assimilate the concepts introduced in the subject
\end{abstract}

Keywords: gamification, e-learning, digital

\begin{abstract}
Resumen
Durante los últimos 3 años se ha observado que el alumnado no tiene una base sólida para entender los conceptos que se introducen en la asignatura. Además, la actitud de los alumnos, en general, es de dejadez, no repasan conceptos para preparar la asignatura, no se preparan las prácticas de laboratorio y dejan de asistir a las clases presenciales. Dando como resultado una alta tasa de abandono, miedo a realizar la asignatura y malestar con el profesorado.

Para resolver esta problemática, se aplica la innovación docente presentada en este artículo. Dicha innovación saca beneficio de estratégias docentes como la gamificación o el aprendizaje basado en problemas, usa herramientas virtuales para motivar el "e-learning" y la colaboración con el resto de estudiantes. Los resultados, hasta la fecha, son satisfactorios dado que reducen la tasa de abandono e incrementan el número de estudiantes que asimilan los conceptos introducidos en la asignatura.
\end{abstract}

Palabras clave: gamificación, e-learning, digital, cuestionarios, prácticas integradas 


\section{Introducción}

"Diseño y síntesis de sistemas digitales" es una asignatura obligatoria impartida durante el $6^{0}$ semestre del grado de Ingeniería Electrónica y de Telecomunicaciones. La asignatura permite cerrar el bloque de enseñanza sobre sistemas digitales dentro la Ingeniería ya que, previamente, el alumno debe haber cursado "Diseño Digital Básico" y "Sistemas Digitales y Estructura de Procesadores" y "Diseño microelectrónico", en los semestres $1^{\circ}, 3^{\circ}$ y $5^{\circ}$ respectivamente.

La metodología formativa que se sigue en la asignatura está dividida en un bloque teórico que ocupa el $40 \%$ de las clases y un bloque práctico que ocupa el $60 \%$ restante. Dentro del bloque teórico, el profesor imparte una clase magistral y hace de moderador en debates sobre textos técnicos que el alumnado debe haber leído previamente. Dentro del bloque práctico, se realizan diversos ejercicios guiados y un proyecto final autónomo que proporcionan las capacidades básicas para ejercer la profesión de ingeniero electrónico en el ámbito del diseño de circuitos digitales.

Aunque no es la continuación de ninguna asignatura troncal, Diseño y Síntesis de Sistemas Digitales sí que se basa en conceptos previamente enseñados en asignaturas de $1^{\circ}, 3^{\circ}$ y $5^{\circ}$ semestre (Diseño Digital Básico, Sistemas Digitales y Estructura de Procesadores y Diseño microelectrónico).

La problemática que observamos los profesores de la asignatura es que los alumnos no tienen una base sólida para entender los conceptos que se introducen en la asignatura. Además, la actitud de los alumnos, en general, es de dejadez, no repasan conceptos para preparar la asignatura, no se preparan las prácticas de laboratorio y dejan de asistir a las clases presenciales.

Las principales consecuencias para el alumnado se pueden resumir como:

- El estudiante no puede seguir el día a día de la asignatura

- El estudiante tiene que hacer un sobreesfuerzo para poder entender los nuevos conceptos

- El alumnado no utiliza las 3 horas de prácticas presenciales para hacer la práctica (debe repasar conceptos).

- El estudiante ve que tiene una carga docente mayor a la que realmente le correspondería a esta asignatura.

- Malestar general por parte del alumnado al creer que la carga docente no es la adecuada.

- Una parte minoritaria del alumnado deja la asignatura por no poder seguir las otras asignaturas.

El profesorado también se ve afectado dado que:

- Se produce una avalancha de alumnos durante las horas de visita del profesorado.

- La actitud del alumnado hacia el profesor, en ocasiones, deja de ser cordial.

- Los debates en las clases de teoría son entre el profesor y una minoría de alumnos

(cc) EY-NC-ND 2017, Universitat Politècnica de València 
- Los profesores en las clases prácticas deben invertir más tiempo aclarando conceptos de asignaturas pasadas.

Delante de esta problemática, el equipo docente de la asignatura se propone implementar una innovación docente basada en el trabajo colaborativo en red, uso de herramientas para la evaluación continuada y debates en grupo. A continuación se describen los antecedentes de la asignatura, los objetivos y desarrollo de la innovación para finalizar presentando los resultados preliminares obtenidos durante este curso y las conclusiones.

\subsection{Antecedentes}

La parte práctica de la asignatura estaba dividida de la siguiente manera:

- Un proyecto (implementación de un sistema de comunicación UART) dividido en 4 partes ( 8 sesiones de laboratorio):

- Introducción (estudio de un sistema de comunicación)

- Diseño de un sistema de comunicación propio (transmisor y receptor)

○ Verificación a nivel funcional (test del sistema mediante simulaciones)

- Implementación física en un sistema real (FPGA)

- Una sesión magistral donde los profesores introducimos como llevar los conocimientos adquiridos a la fabricación de chips (como por ejemplo los microprocesadores Intel) (1 sesión de laboratorio)

- Un proyecto final individual que el alumno realiza fuera del entorno de prácticas. El alumnado tenía un mes desde la propuesta del proyecto hasta el día de entrega.

Anteriormente el equipo docente ya había iniciado diversas actuaciones:

- Iniciar los laboratorios dos semanas antes para introducir/repasar los conceptos necesarios para comenzar las prácticas.

- Cuestionarios tipo test obligatorios antes de entrar al laboratorio para hacer que el alumno lea las prácticas. Estos cuestionarios afectan a la nota final y se hacen durante los primeros 20 minutos de cada práctica para obligar al alumno a ser puntual.

- La evaluación de cada práctica es diferente si el alumno la ha entregado en la fecha de entrega o a posteriori. Si el alumno hace la entrega antes de la fecha límite su nota puede ir de 0 a 10 . Si el alumno la entrega fuera del límite, la máxima nota será un 5. Si por algún motivo la práctica del alumno no está aprobada, el alumno tiene tantas oportunidades como sean necesarios de volver a presentarla hasta que alcance el 5.

Los resultados de estas actuaciones se pueden calificar de malos ya que:

- El alumnado motivado se aburre durante las prácticas introductorias. Las prácticas tienen una duración de 3 horas y se está volviendo habitual ver este tipo de alumno navegando por internet (facebook, diarios online, ...) y no realizando la práctica. 
- La mayoría del alumnado llega sin leerse la práctica y parece no tener ningún problema en suspender el cuestionario, el número máximo de cuestionarios aprobados es de 6 sobre 30 alumnos). Además, continuamos viendo alumnos que llegan más de 30 minutos tarde, a los que parece que tampoco afecta tener un 0 al cuestionario.

- El hecho de tener la opción de entregar más tarde la práctica ha hecho que más de un $60 \%$ de los alumnos las entregue el último día de clase.

\section{Objetivos}

En general, aunque el alumnado suele haber demostrado que alcanza los conocimientos mínimos de cada asignatura previa, hemos detectado que el alumnado, ya sea por olvido, dejadez o el itinerario que han seguido en el grado, demuestra no tener el nivel mínimo al comienzo de la asignatura.

Por lo tanto, el objetivo de la innovación es pasar a un sistema de prácticas integradas donde inicialmente se evaluará el nivel inicial de cada alumno y se le acompañará, a través de las prácticas, durante el resto del curso para alcanzar el nivel final requerido. En consecuencia, pretendemos asegurar que el alumno pueda realizar el curso, con el nivel de exigencia requerido, sin que tenga que dedicar un sobre esfuerzo debido a su falta de conocimientos o falta de interés. Para alcanzar estos objetivos se plantean diversas actuaciones:

- Hacer uso del entorno "Moodle" para generar cuestionarios en línea para motivar que el alumno repase los conceptos previos a la asignatura, detectar su nivel y guiarlo para alcanzar los conceptos requeridos, preparar las sesiones de discusión técnicas en clase y obligar al alumno a preparar las prácticas antes de la clase presencial.

- Hacer uso del entorno "Moodle" y "Wikispaces" moderado por el profesor para generar lo que se llama «e-learning» colaborativo. Con estas herramientas el alumno pasa a ser parte activa en el aprendizaje ya que puede proponer, solucionar o preguntar dudas. El hecho de que el moderador sea el profesor asegura que el resultado final siempre será el adecuado y que lo verán todos los alumnos. Así pues, el profesor tendrá que dedicar menos horas de visita a solucionar dudas.

- Hacer uso del entorno "Moodle" para motivar el trabajo colaborativo en red para aquellas prácticas que puedan hacer en grupo.

- Uso de estratégias docentes como "aprendizaje basado en problemas" y "gamificación" para la realización del proyecto final. Por ejemplo, proponiendo proyectos finales que consistan en la implementación de juegos en FPGAs.

(c) EY-NC-ND 2017, Universitat Politècnica de València 


\section{Desarrollo de la innovación}

Lo que hacía el equipo docente durante las sesiones prácticas de la asignatura era preparar al alumno para poder realizar un proyecto de manera autónoma. Para motivar al alumno, estas sesiones prácticas también tenían incidencia en la nota final.

Para que el equipo docente haya transformado estas prácticas compartimentadas en prácticas integradas han sido necesarias varias actuaciones:

- Buscar el nivel del alumno durante el inicio de la primera práctica. La metodología empleada es un cuestionario con preguntas de respuesta abierta, Cierto / Falso y con preguntas de múltiples opciones.

- Agrupar a los alumnos en 4 niveles diferentes (de hasta 20 alumnos) según los resultados del test de nivel inicial. A partir de este punto adaptamos los contenidos a cada grupo para que todos puedan llegar al final del curso con el nivel requerido. Por lo tanto, generamos y fomentamos el uso de material docente de refuerzo, de obligada lectura y adaptado al nivel de cada grupo para alcanzar el nivel mínimo requerido para realizar la práctica. Además, los grupos de nivel más alto se les puede motivar con ejemplos y aplicaciones más avanzadas.

- Con el fin de motivar a los alumnos a realizar los cuestionarios y las prácticas hemos cambiado la forma de evaluarlos. Hasta la aplicación de la innovación, tanto los cuestionarios como las prácticas contaban en un pequeño porcentaje en la nota final. Lo que se ha aplicado es cambiar el concepto de práctica puntuable al concepto de práctica necesaria para el proceso de aprendizaje. Así pues, a aplicamos el siguiente sistema de evaluación: si el cuestionario o práctica está correcto, la nota es un 0 . Si por el contrario está suspendida, la nota es -1 . Si se ha realizado el correcto aprendizaje, la nota del proyecto final no queda penalizada por la nota de prácticas. Por el contrario, si no se ha llevado a cabo el proceso de aprendizaje, la nota final del alumno sí quedará penalizada. Dicho de otra manera, el equipo docente pretende fomentar la realización de los cuestionarios y las prácticas y pretende penalizar en la nota final el no haber realizado las actividades.

- Uso del entorno "Moodle" para generar cuestionarios en línea de respuesta abierta para motivar que el alumno prepare las sesiones de discusión técnicas en clase y obligar al alumno a preparar las prácticas antes de la clase presencial. Estos cuestionarios son obligatorios y se realizan antes de la realizar los debates. Esto Por también permite conocer las carencias de cada alumno y, por tanto, permite hacer una actuación sobre cada alumno para que alcance los conceptos mínimos requeridos.

- Uso del entorno "Moodle" y "Wikispaces" moderado por el profesor para generar lo que se llama «e-learning» colaborativo. Con estas herramientas el alumno es parte activa en el aprendizaje ya que tanto puede proponer actividades a los compañeros como solucionar o preguntar dudas. El hecho de que el moderador sea el profesor asegura que el resultado final siempre es el adecuado. De esta manera, el profesor dedica menos horas de visita para solucionar dudas. De igual manera, 
permite al alumno realizar el aprendizaje de manera autónoma y, como utiliza herramientas virtuales, el estudiante se desinhibe a la hora de resolver dudas.

La innovación en este punto reside en que el alumnado puede preguntar dudas y también puede contestar las dudas de otros compañeros. Si esto no sucede, el equipo docente es el encargado de provocar/fomentar la participación de los alumnos. En un futuro próximo, el equipo docente tendrá todos los datos necesarios para generar un documento de "preguntas frecuentes" que habrá sido generarado por los propios alumnos.

- Uso del entorno "Moodle" para motivar el trabajo colaborativo en red para aquellas prácticas que puedan hacer en grupo. La innovación reside en hacer que la mitad de cada grupo realice un transmisor y la otra mitad realice un receptor para fomentar la colaboración (para que el sistema final funcione, los alumnos deben utilizar el sistema de comunicación complementario de otro compañero). El principal beneficio es la mejora del trabajo en equipo de los alumnos ya que no nos encontramos con las típicas excusas como "no tenía tiempo de quedar con los compañeros" o "no le hemos dicho nada a la otro compañero porqué no nos cae bien".

En resumen, la innovación docente ha adaptado la asignatura a una serie de prácticas integradas donde se evalua el nivel de cada alumno mediante una serie de cuestionarios creados especialmente para esta tarea y, actuamos sobre cada alumno a través de material docente extra y el uso de herramientas «online» que permiten el aprendizaje colaborativo y acompañar al alumno hasta alcanzar el nivel requerido.

\section{Resultados}

La implementación de la innovación docente se está llevando a cabo durante este curso 2016-17 (semestre de primavera). Los resultados que presentamos aquí son provisionales, dado que sólo podemos comparar el numero de abandonos que ha habido hasta la mitad de las prácticas. Tabla 1 presenta los detalles de alumnos matriculados, abandonos (no presentados más des-matriculados), numero de aprobados (dos convocatorias) y suspensos de la asignatura durante los años previos a la implementación de la innovación y a partir de ella.

Tabla 1. Num. De alumnos matriculados, abandonos, aprobados y suspensos por curso

\begin{tabular}{ccccc}
\hline Alumnos & $\mathbf{2 0 1 3}$ & $\mathbf{2 0 1 4}$ & $\mathbf{2 0 1 5}$ & $\mathbf{2 0 1 6}$ \\
\hline Matriculados & 17 & 24 & 27 & 28 \\
Abandonos & 9 & 10 & 15 & $3 *$ \\
Aprobados & 11 & 17 & 12 & \\
Suspendidos & 2 & 1 & 0 & \\
\hline
\end{tabular}

(cc) EY-NC-ND 2017, Universitat Politècnica de València 
*Datos provisionales

Los indicadores de evaluación que esperamos que determinen si el alumnado ha mejorado son los siguientes:

- Reducción de errores de conceptos básico a los exámenes. Hasta la aplicación de la innovación, entre un $15 \%$ y $20 \%$ de los alumnos matriculados no terminaba el curso sabiendo realizar las estructuras mínimas enseñadas a principio de este. Esperamos reducir este porcentaje al 2\% o 3\%.

- Disminución de la tasa de abandono.

- Reducción del esfuerzo dedicado del alumno. Actualmente el alumnado no encuentra que el esfuerzo de la asignatura se adecue a los créditos de esta (6 creditos) y, según las quejas del alumnado, la asignatura debería tener "muchos más" créditos. Esperamos reducir este esfuerzo para que el alumno tenga la percepción real de las horas dedicadas a la asignatura. Los resultados los mediremos con las encuestas, donde se mide el tiempo real dedicado a la asignatura.

- Tasa de aprobados y aumento del número de alumnos presentados

\section{Conclusiones}

La innovación docente presentada pretende medir y cuantificar de manera objetiva los resultados sobre el estudiante a través de las acciones indicadas previamente. Es por eso que como resultado final se obtendrá:

- Tiempo real que ha dedicado cada estudiante en la asignatura. Esto se obtendrá a través de las encuestas realizadas durante el curso. De esta manera el esfuerzo del alumnado deja de ser una percepción.

- Medida de la tasa de abandono.

- Logro por parte del alumnado del nivel final requerido para aprobar.

- Mejora en la realización de las prácticas.

Durante la aplicación de la innovación docente hemos observado que, de momento, la tasa de abandono se ha reducido, aunque no nula. Además, la implicación del alumnado es más alta: se preparan las prácticas, trabajan en el laboratorio (sólo en un par de casos excepcionales se les ha tenido que llamar la atención por no estar trabajando), el $85 \%$ aprueba sin problemas los cuestionarios (de los suspendidos la mitad lo hace con un 4 o más), casi el $80 \%$ de los alumnos ha hecho una propuesta de proyecto.

El único problema que, a día de hoy, observamos es la baja participación nos los foros. La mayoría de las participaciones han sido promovidas por el equipo docente. 
Mejora de la implicación del alumnado en "Diseño y sintesis de sistemas digitales" usando e-learning colaborativo, gamificación y aprendizaje basado en problemas

\section{Referencias}

FONT, A. (2004) "Líneas maestras del Aprendizaje por Problemas", Revista Interuniversitaria de

Formación del Profesorado. vol. 18, num. 1, p. 79-92.

MICHAELSEN, L.K.; SWEET, M. (2009) "The essential elements of team-based learning", Team-Based Learning: Small Group Learning's Next Big Step. New Directions in Teaching and Learning, p. 7-27.

KAPP, K. (2012) The gamification of learning and instruction. Game-based methods and strategies for training and education. San Francisco: Pfeiffer-Wiley.

KHAIRY, B. (2015) "The Standards of Intelligent Collaborative e-Learning of e-Courses Based on Web”, Conference on e-Learning (econf), 2015 Fifth International, p. 486-493.

WILLEY, K. (2016) "Combining a collaborative learning framework with an e-learning tool to improve learning and professional development in blended learning environments", Future Technologies Conference (FTC), p. 1303-1304.

(c) EY-NC-ND 2017, Universitat Politècnica de València 